Original Article

\title{
Effects of dry needling on tendon-pulley architecture, pain and hand function in patients with trigger finger: a randomized controlled trial study
}

Morteza Azizian, PT ${ }^{1)}$, Hossein Bagheri, PhD, $\left.\mathrm{PT}^{2}\right)^{*}$, Gholam Olyaei, PhD, $\mathrm{PT}^{2)}$, Azadeh Shadmehr, PhD, PT ${ }^{2)}$, Mohammad Ali Okhovatpour, MD ${ }^{3)}$, Pooneh Dehghan, MD $^{4)}$, Shohreh Jalaei, PhD ${ }^{5)}$, Hadi Sarafraz, PhD, PT $^{6}$ )

1) Department of Physiotherapy, School of Rehabilitation, International Campus, Tehran University of Medical Sciences, Iran

2) Department of Physiotherapy, School of Rehabilitation, Tehran University of Medical Sciences: Tehran 11155-1683, Iran

3) Department of Orthopedics Hand Sugery, Taleghani Hospital, Shahid Beheshti University of Medical Sciences, Iran

4) Department of Radiology, Taleghani Hospital, Shahid Beheshti University of Medical Sciences, Iran

5) Department of Physiotherapy, School of Rehabilitation, Tehran University of Medical Sciences, Iran

6) Department of Orthopedic surgery, Shahid Mohammadi Hospital, Hormozgan University of Medical Sciences, Iran

\begin{abstract}
Purpose] The purpose of this study was to determine the effect of dry needling on tendon-pulley architecture, pain and hand function in patients with trigger finger. [Participants and Methods] A randomized controlled trial was conducted. Fifty eight patients having trigger finger were randomly assigned as either an experimental group that received a single session of dry needling over pulleyA1 and flexor tendon or a control group that received no intervention. Thickness of tendon-pulley, and pain-hand function (by disability arm-shoulder questionnaire score and pinch grip strength) were measured by a blinded assessor before and one week after intervention. [Results] The two way mixed ANOVA in the experimental group showed that the thickness of pulley-tendon decreased, pinch grip power increased and DASH questionnaire score was decreased in comparison to the control group. [Conclusion] This study results suggest that a single session of Dry Needling (DN) was effective in decreasing pain, DASH score, pulley-tendon thickness and improving pinch grip power in patients with trigger finger. Keywords: Trigger finger, Dry needling, Pulley-tendon architecture
\end{abstract}

(This article was submitted Jul. 14, 2018, and was accepted Oct. 24, 2018)

\section{INTRODUCTION}

Trigger finger (TF) is one of the most prevalent causes of hand disability and is a common cause of referral to orthopedic clinics $^{1)}$. The incidence of TF is $28: 100,000$ per year or a lifetime risk of $2.6 \%$ in the general population ${ }^{2}$. Although trigger finger is considered a mild hand pathology, it has a wide-ranging impact on hand functioning, daily activities and quality of life ${ }^{3}$. It can be caused by inflammation and subsequent narrowing of the A1 pulley and flexor tendon thickening, causing pain, clicking, catching, and loss of motion of the affected finger ${ }^{4}$.

*Corresponding author. Hossein Bagheri (E-mail: hbagheri@.tums.ac.ir)

(C2019 The Society of Physical Therapy Science. Published by IPEC Inc.

(c) (1) $($ This is an open-access article distributed under the terms of the Creative Commons Attribution Non-Commercial No Derivatives cC. 
Different clinical interventions are used to treat trigger finger ranging from conservative to surgical procedure. Surgical treatments are invasive and have potential complications ${ }^{5}$. Conservative treatment includes rest and avoidance of gripping activities, as well as activity modification, ice and physical therapy ${ }^{6}$. Recent meta-analysis showed that injection may be an effective strategy to manage trigger finger ${ }^{7}$. Dry needling (DN) is a relatively new technique used by physical therapists to treat myofascial trigger points (MTrPs) and various pain syndromes.

Dry needling requires insertion of thin monofilament needles, as used in the practice of acupuncture, without using injection into muscles, ligaments, tendons, subcutaneous fascia, and scar for pain management and functional improvement ${ }^{8}$.

To the best of our knowledge, no previous study has investigated the effects of dry needling on tendon-pulley morphology and hand function in patients with trigger finger. Therefore, the main aim of this study was to assess short term effects of dry needling on tendon-pulley architecture, pinch grip, DASH score and pain in groups of patients.

\section{PARTICIPANTS AND METHODS}

This randomized controlled trial study was conducted from February 2017 to May 2018 for twenty weeks. The study was approved by Iranian clinical trials review boards (IRCT2015101024453N1). Prior to study participants signed a consent form. Fifty eight participants aged 45-75 years who were diagnosed with trigger finger with grade 1, 2, 3 according to quinnell classification (Grade 1: Uneven movement, Grade 2: Actively correctable, Grade 3: Passively correctable, Grade 4: Fixed deformity during finger flexion and extension) were selected ${ }^{9}$. The participants were included if they had (1) unilateral idiopathic trigger finger lasting at least 4 weeks (2) pain and tenderness at the position of A1 pulley, nodule palpation in this zone, pain and discomfort when flexing and extending the finger, nodule palpation, presence of a clicking sound at the time of flexion or extension of the finger, snapping or locking of finger, and the existence of a trigger state. Participants with diabetes mellitus, history of trauma, rheumatoid arthritis, dialysis treatment, fingers with a history of local gouty/pyogenic disease, or major hand trauma and fear of needle and any contraindication for deep dry needling such as anticoagulants, infections, bleeding, or psychotic conditions tumors, calcium deposits, or severe osteoarthritis were excluded.

Fifty eight participants with trigger finger were sampled and randomly (group names picked from a bowl) assigned to two groups. Sample size fifty eight was calculated based on a pilot study by using G power software package. Twenty nine participants were assigned to intervention group and twenty nine controls without intervention. Orthopedic surgeon administered the trigger finger glove for affected hand for preventing of exercises in both groups.

A single session of dry needling with disposable stainless steel needles $(0.3 \times 30 \mathrm{~mm}$; Dong Bang, Korea $)$ inserted into the skin over nodule in A1 pulley anatomic location. In this study, the fast-in and fast-out technique described by Hong ${ }^{10)}$ was applied. Duration of needling was 1 minute. The needle was inserted deeply $45^{\circ}$ to the level of metacarpophalangial level to nodule using the fast-in and fast-out cone shape technique. The needle may be inserted into the tendon. This is confirmed by needle movement when the patient flexes and extends the distal phalanx. The needle is withdrawn slowly until this motion ceases. The needle tip is now in the A1 pulley. The experienced trained (Therapist with 5 years dry needling practice with license acupuncture course) performed dry needling of the muscles was blinded to the outcome measurements. Before intervention, pain DASH score, pully-tendon thickness and pinch grip were measured. One week later, these outcomes were measured using the same method. Pain and pinch grip were measured by visual analog scale and digital hand dynamometer. The DASH is a 30-item questionnaire measuring the patient's functional status during the preceding week. Tendon-pully architecture was measured using an ultrasonography device (SamsungWS80A-Korea) by sonographer specialist in musculoskeletal disorders. Fingers were evaluated under real-time B-mode at a frequency of $12 \mathrm{MHz}$ and at a depth of $3 \mathrm{~cm}$. Sonographic examination was performed with the forearm supinated, wrist in neutral position and MP joint in extension. The probe was positioned perpendicular to the palmar surface at the MP joint. A1 pully thickness and tendon cross-section area in axial view were measured.

Kolmogorov-Smirnov test showed normal distribution of all variables. A $2 \times 2$ mixed model repeated-measure analysis of variance (ANOVA) with time (baseline, one week after) as the within-participant factor, group (no intervention, dry needling) as the between-participants factor, was used to determine the effects of the treatment on pain, pinch grip, DASH, Tendon-pulley thickness. SPSS version 22.0 and the signifcane level set at $\alpha=0.05$ as well as.

\section{RESULTS}

Seventy consecutive patients who had trigger finger (Grade 1, 2, 3) were screened for eligibility. Fifty-eight satisfied the eligibility criteria, agreed to participate, and were randomized into the exprimental group ( $\mathrm{n}=29$, mean \pm SD age 60.24 \pm 8.54 years; $55 \%$ female) or the control group ( $\mathrm{n}=29$, mean \pm SD age $62.00 \pm 7.73$ years; $62 \%$ female). Baseline features between both groups were similar for all outcomes. Demographic data of participants are shown in Table 1. Comparison between group revealed significant group $\times$ time for VAS score, hand function and pulley tendon morphology as summarized in Table 2. According to Table 2, There was a significant difference within experimental group but no difference in control group. 
Table 1. Baseline demographic and clinical outcomes

\begin{tabular}{lccc}
\hline \multicolumn{1}{c}{ Clinical feature } & $\begin{array}{c}\text { Experimental group } \\
(\mathrm{n}=29)\end{array}$ & $\begin{array}{c}\text { Control group } \\
(\mathrm{n}=29)\end{array}$ \\
\hline Gender (male/female) & & $13 / 16$ & $11 / 18$ \\
Age (years) & & $60.24 \pm 8.54$ & $62.00 \pm 7.73$ \\
Trigger finger grade & I & 1 & 5 \\
& II & 20 & 18 \\
& III & 8 & 6 \\
\hline
\end{tabular}

Table 2. Outcomes measurement of pre-intervention and one week after intervention

\begin{tabular}{|c|c|c|c|c|c|c|c|}
\hline & \multicolumn{2}{|c|}{ Experimental $(n=29)$} & \multicolumn{2}{|c|}{ Control $(n=29)$} & \multicolumn{3}{|c|}{$\mathrm{p}$ value } \\
\hline & Before & After & Before & After & Time & Group & Interaction \\
\hline VAS (scores) & $4.43 \pm 0.90$ & $1.79 \pm 0.54$ & $3.83 \pm 0.88$ & $3.59 \pm 0.90$ & * & $*$ & $*$ \\
\hline DASH (scores) & $23.81 \pm 7.3$ & $19.24 \pm 8.57$ & $20.66 \pm 7.71$ & $20.91 \pm 7.48$ & $*$ & $*$ & $*$ \\
\hline Pinch grip (kg) & $4.6 \pm 1.53$ & $5.40 \pm 1.45$ & $4.67 \pm 1.44$ & $4.63 \pm 1.50$ & $*$ & * & $*$ \\
\hline A1 pulley $(\mathrm{mm})$ & $1.19 \pm 0.30$ & $0.97 \pm 0.33$ & $1.22 \pm 0.31$ & $1.25 \pm 0.28$ & $*$ & $*$ & $*$ \\
\hline Tendon thickness (mm) & $3.91 \pm 0.58$ & $3.78 \pm 0.79$ & $4 \pm 0.62$ & $4.02 \pm 0.81$ & $*$ & $*$ & $*$ \\
\hline
\end{tabular}

Statistical significant differences (ANOVA repeated measure two way $2 \times 2$, group $\times$ time, $(\mathrm{p}<0.05)$ ).

\section{DISCUSSION}

The current randomized controlled trial is the first one to analyze the effects of dry needling on hand performance and tendon-pulley morphology in patients with trigger finger. A significant decrease of pain one week after intervention could be due to the central effect of dry needling (i.e, activation of various sensory pathways and noxious inhibitory control system resulted in neuromodulation of pain signaling ${ }^{8}$.

Dry needling activates spinal segmental pain inhibitory and descending pain control pathways. Needle manipulation stimulates the release endogenous opioids, which is considered one of the most potent mechanisms for pain suppression in the periphery and spinal cord level secondary to needling treatment ${ }^{11,12)}$.

The study results showed that thickness of tendon-pulley decreased one week after dry needling. By using an axial highresolution ultrasonography after a single session of dry needling for involved digit, not only the thickness of the pulley, but also the volume of the tendon were reduced and clinical symptoms were improved. There were no studies to analyze these parameters after dry needling in trigger finger patients. Hence, we can compare our results with the studies on various injection solutions such a betametazon and triamcinolone acetonide in trigger finger patients. Our results are in agreement with those obtained by Takahashi et al. who also reported a decrease of A1 pulley thickness and tendon volume (cross section) in the long term. However, the current improvement in our clinical trial study was observed in the short term, while betametazon injection was showed with a peak effect in three months ${ }^{13)}$.

Consequently, different factors could possibly be considered in injection of corticosteroid and dry needling effects on pulley tendon morphology ${ }^{13,14}$. These findings can interpret the reduction of pully thickness and tendon volume as a major mechanism, which dry needling affects trigger finger. In contrast to the tendon consisting of a dense collagen matrix, the pulley has been shown to have a loose connective layer in addition to a dense normal connective layer ${ }^{15}$.

These less dense elements are regarded to be more susceptible to dry needling than dense tissues such as tendons. Furthermore, dry needling can affect tendon sheath rather than tendon due to less density of connective tissue. Therefore, gliding of flexor tendon/tendon movement in tendon sheath improved after dry needling.

Regarding the short time effect of dry needling on hand function (DASH score, Pinch grip), significant decrease in DASH score and improvement in pinch grip strength were found. These results could be owing to decrease of pain and at least one grade improvement in trigger finger grade was achieved in this study group by the time of the second examination. This was in line with the result reported by Dogru et al. concerning decrease of pain severity, improvement of general functional capacity (DASH Score) and pinch strength in patients with trigger finger after shock wave therapy ${ }^{16)}$.

Although the results of our randomized controlled trial are promising, potential limitations should be recognized. In this study, only one treatment session in the short term period was used with dry needling. In this regard, future studies should include multiple treatment sessions with a greater number of clinicians and longer follow-up periods.

The results of the present study suggest that one session of dry needling into trigger finger patients was effective in decreasing pain and improving hand functionality (DASH score, pinch grip strength) by reducing A1 pulley thickness and tendon volume (cross section area). 


\section{Funding}

This paper was supported by the International Campus, Tehran University of Medical Sciences (IC-TUMS).

Conflict of interest

None.

\section{ACKNOWLEDGEMENT}

The authors would like to acknowledge the generous assistance staff of the Sonography Unit of Taleghani Hospital, Shahid Beheshti University of Medical Sciences, Tehran, Iran.

\section{REFERENCES}

1) Mardani Kivi M, Lahiji FA, Jandaghi AB, et al.: Efficacy of sonographically guided intra-flexoral sheath corticosteroid injection in the treatment of trigger thumb. Acta Orthop Traumatol Turc, 2012, 46: 346-352. [Medline] [CrossRef]

2) Akhtar S, Bradley MJ, Quinton DN, et al.: Management and referral for trigger finger/thumb. BMJ, 2005, 331: 30-33. [Medline] [CrossRef]

3) Langer D, Maeir A, Michailevich M, et al.: Using the international classification of functioning to examine the impact of trigger finger. Disabil Rehabil, 2016, 38: 2530-2537. [Medline] [CrossRef]

4) Makkouk AH, Oetgen ME, Swigart CR, et al.: Trigger finger: etiology, evaluation, and treatment. Curr Rev Musculoskelet Med, 2008, 1: 92-96. [Medline] [CrossRef]

5) Cebesoy O, Kose KC, Baltaci ET, et al.: Percutaneous release of the trigger thumb: is it safe, cheap and effective? Int Orthop, 2007, 31: 345-349. [Medline] [CrossRef]

6) Vasiliadis AV, Itsiopoulos I: Trigger finger: an atraumatic medical phenomenon. J Hand Surg Asian Pac Vol, 2017, 22: 188-193. [Medline] [CrossRef]

7) Ma S, Wang C, Li J, et al.: Efficacy of corticosteroid injection for treatment of trigger finger: a meta-analysis of randomized controlled trials. J Invest Surg, 2018, 30: 1-9. [Medline] [CrossRef]

8) Dunning J, Butts R, Mourad F, et al.: Dry needling: a literature review with implications for clinical practice guidelines. Phys Ther Rev, 2014, 19: 252-265. [Medline] [CrossRef]

9) Quinnell RC: Conservative management of trigger finger. Practitioner, 1980, 224: 187-190. [Medline]

10) Hong CZ: Lidocaine injection versus dry needling to myofascial trigger point. The importance of the local twitch response. Am J Phys Med Rehabil, 1994, 73: 256-263. [Medline] [CrossRef]

11) Niddam DM, Chan RC, Lee SH, et al.: Central modulation of pain evoked from myofascial trigger point. Clin J Pain, 2007, 23: 440-448. [Medline] [CrossRef]

12) Hsieh YL, Chou LW, Joe YS, et al.: Spinal cord mechanism involving the remote effects of dry needling on the irritability of myofascial trigger spots in rabbit skeletal muscle. Arch Phys Med Rehabil, 2011, 92: 1098-1105. [Medline] [CrossRef]

13) Takahashi M, Sato R, Kondo K, et al.: Morphological alterations of the tendon and pulley on ultrasound after intrasynovial injection of betamethasone for trigger digit. Ultrasonography, 2018, 37: 134-139. [Medline] [CrossRef]

14) Miyamoto H, Miura T, Isayama H, et al.: Stiffness of the first annular pulley in normal and trigger fingers. J Hand Surg Am, 2011, 36: 1486-1491. [Medline] [CrossRef]

15) Sbernardori MC, Bandiera P: Histopathology of the A1 pulley in adult trigger fingers. J Hand Surg Eur Vol, 2007, 32: 556-559. [Medline] [CrossRef]

16) Dogru M, Narin S, Erduran M: SAT0594 The effect of radial extracorporeal shock wave therapy (RESWT) in the treatment of trigger finger. Ann Rheum Dis, 2017, 76: 999. 